PERSPECTIVE

\title{
Capillary flow of liquids in open microchannels: overview and recent advances
}

\author{
Panayiotis Kolliopoulos (iD) and Satish Kumar (iD ${ }^{1 凶}$
}

Capillary flow is the spontaneous wicking of liquids in narrow spaces without the assistance of external forces. Examples of capillary flow can be found in numerous applications ranging from controlling and transporting fuel in spacecrafts to printed electronics manufacturing. Open rectangular microchannels often appear in these applications, with the lack of a top resulting in a complex free-surface morphology and evaporation. Here, we present a brief overview of this topic and discuss some recent advances.

npj Microgravity (2021)7:51; https://doi.org/10.1038/s41526-021-00180-6

\section{INTRODUCTION}

Capillary flow is the spontaneous wicking of liquids in narrow spaces without the assistance of external forces. This phenomenon is common in everyday life and industrial applications. Examples of capillary flow can be found in diverse fields, ranging from physiology, where capillary flow is essential for the drainage of tear fluid from the eye, to flexible printed electronics manufacturing $^{1-3}$, where capillary flow is used to create electronic circuits that can be used in sensors for brain-machine interfacing. Additional applications include lab-on-a-chip devices ${ }^{4,5}$, heat pipes $^{6}$, propellant management devices in spacecrafts ${ }^{7}$, paperbased microfluidics ${ }^{8}$, fuel cells ${ }^{9}$, porous-media flows ${ }^{10,11}$, and storage and handling of fluids and waste in low-gravity environments ${ }^{12,13}$. Understanding the mechanism of capillary flow and finding ways to control it has been a subject of investigation since the early twentieth century.

The purpose of this Perspective article is to provide a brief overview of and discuss some recent advances in our fundamental understanding of capillary flow for an important special case: rectangular microchannels without a top. Such open microchannels appear in a variety of applications and involve more complex flow behavior than their closed counterparts. In addition, the liquid in the microchannel may evaporate, leading to further challenges in understanding flow behavior. Notably, the channels involved have widths and depths of tens to hundreds of microns so that surface tension, i.e., capillary forces play a key role in driving flow. The importance of capillary flow and evaporation in open microchannels along with recent insights obtained through an interplay between theory and experiment ${ }^{14,15}$ make this Perspective article particularly timely, and we hope it will inspire others to advance both the fundamentals and applications related to this topic. We first discuss capillary-flow dynamics in the absence of liquid evaporation, and then consider the influence of evaporation.

\section{CAPILLARY-FLOW DYNAMICS}

Early studies by Lucas ${ }^{16}$ and Washburn ${ }^{17}$ focused on developing theoretical models to understand the physical mechanisms driving spontaneous capillary flow in cylindrical tubes. Their models described the meniscus position $\hat{z}_{M}$ as a function of time $\hat{t}$ for flow of Newtonian liquids. Lucas ${ }^{16}$ assumed the flow is driven by the capillary pressure gradient caused by the circular-arc meniscus front, while Washburn ${ }^{17}$ also included hydrostatic pressure gradients and an imposed pressure difference between the two ends of the cylindrical tube. For a horizontal cylindrical tube open at both ends, an analytical solution $\hat{z}_{M}=\sqrt{\hat{k} \hat{t}}$ is obtained, commonly referred to as the Lucas-Washburn relation, where $\hat{k}$ is the mobility parameter and depends on the cylinder radius, liquid viscosity $\hat{\mu}$, surface tension $\hat{\sigma}$, and contact angle $\theta_{0}$. The mobility parameter can be thought of as a diffusion coefficient driving the growth of the liquid interface.

Numerous investigators extended the theoretical work of Lucas $^{16}$ and Washburn ${ }^{17}$ by including inertial ${ }^{18-20}$, gravitational ${ }^{21}$, dynamic contact angle ${ }^{22-24}$, surface roughness ${ }^{24,25}$, wettability gradient $^{26}$, electrowetting ${ }^{27}$, and non-Newtonian rheology ${ }^{28,29}$ effects. In addition, these theoretical models have been extensively compared to experiments ${ }^{18,19,23,24,30-34}$. Prior studies have identified two characteristic regimes for capillary flow in horizontal capillaries, where gravitational forces play a negligible role in driving flow. At early stages the flow is dominated by inertial effects, resulting in $\hat{z}_{M} \sim \hat{t}$. At later stages, inertial effects are negligible, and viscous effects dominate, resulting in $\hat{z}_{M} \sim \hat{t}^{1 / 2}$. Both scalings have been confirmed experimentally.

For vertical or inclined capillaries, gravitational forces can have a significant influence on the flow. As a result, at later times a third characteristic regime is observed where gravitational effects dominate, leading to an asymptotic plateau in $\hat{z}_{M}$. In general, the relative importance of gravitational forces to surface-tension forces is given by the Bond number, Bo $=\hat{\rho} \hat{g} \hat{D}^{2} / \hat{\sigma}$, where $\hat{\rho}$ is the liquid density, $\hat{g}$ is the magnitude of the gravitational acceleration, and $\hat{D}$ is a characteristic length scale. The discussion in this article is primarily focused on the case of vanishing Bo, where gravitational effects are negligible.

Microchannels can broadly be classified as closed (Fig. 1a) or open (Fig. 1b). A closed microchannel is defined as one where all walls are solid, whereas an open microchannel lacks a top. Due to breakthroughs in lithographic fabrication techniques, open microchannels with various cross-sectional geometries (Fig. 1b-d) can be fabricated easily and inexpensively, including rectangular ${ }^{14,15,35-37}$, trapezoidal ${ }^{38}$, U-shaped ${ }^{35}$, and V-shaped ${ }^{39-43}$ cross-sections. The lack of a top provides access to the inside of the channel and has been exploited in applications such as capillary micromoulding

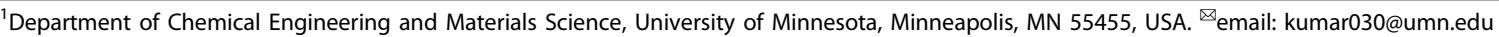


(a)

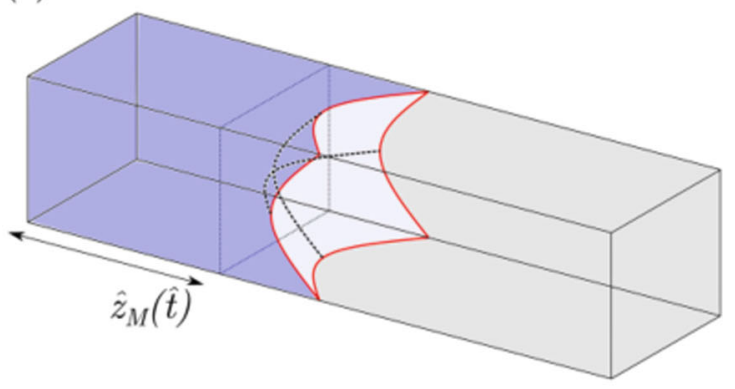

(c)

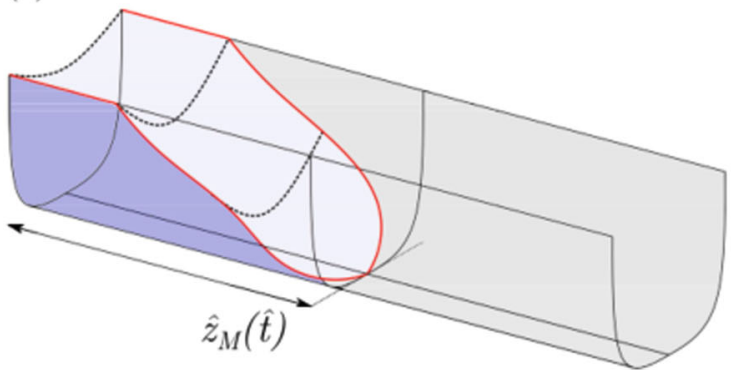

(b)

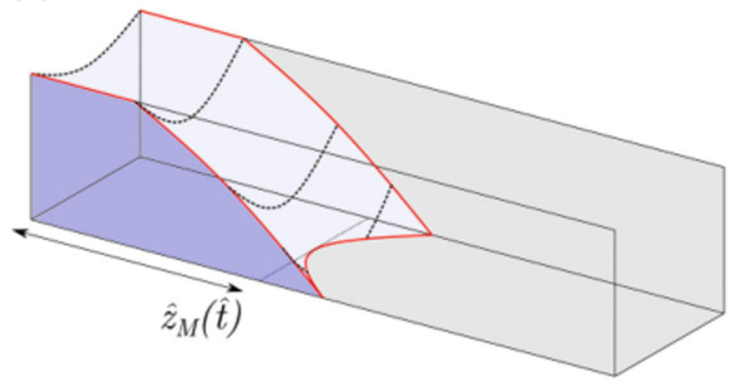

(d)

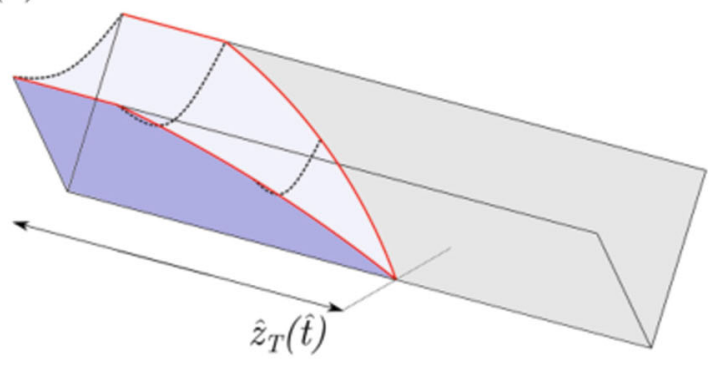

Fig. 1 Microchannel shapes. Schematic of flow in (a) closed rectangular, (b) open rectangular, (c) open U-shaped, and (d) open V-shaped microchannels.

and microfluidics. In addition, the risk of clogging in open microchannels is reduced compared to their closed counterparts ${ }^{5}$.

Motivated by the advantages of open microchannels, investigators have examined capillary flow in these geometries using theory and experiment. Many studies modified the Lucas-Washburn relation, which was initially developed for cylindrical tubes, to model arbitrary cross-sectional geometries ${ }^{24,44}$. In these studies, while the scaling of $\hat{z}_{\mathrm{M}} \sim \hat{t}^{1 / 2}$ still holds, the expression for the mobility parameter $\hat{k}$ depends on the crosssectional geometry. However, predictions from these modified Lucas-Washburn models (MLWs) for open channels have resulted in a varying agreement with experiments ${ }^{14,15,24,35,36,38}$. This is because the mechanism for capillary flow in open channels is more complex than that for closed channels. While for closed channels the force driving the flow is due to the pressure gradient caused by the circular-arc meniscus front (Fig. 1a), for open channels the additional free surface due to the lack of a top also contributes to driving the flow (Fig. 1b-d) ${ }^{15}$.

The additional contribution of the free-surface curvature to capillary flow has been theoretically and experimentally investigated primarily for V-shaped channels ${ }^{39-42,45,46}$. Spontaneous flow in V-shaped channels is observed if the liquid contact angle satisfies $\theta_{0}<\pi / 2-\beta$, as described by Concus and Finn ${ }^{47}$, where $\beta$ is the half-angle of the interior corner. The flow leads to the formation of filaments or fingers, where in horizontally oriented channels or in low-gravity environments the finger tip $\hat{z}_{T}$ scales as $\hat{Z}_{\mathrm{T}} \sim \hat{t}^{1 / 2} 39-42,45,46,48$ and in vertically oriented channels in the presence of gravity it scales as $\hat{z}_{\mathrm{T}} \sim \hat{t}^{1 / 349,50}$.

The most widely used open-channel cross-sectional geometry is rectangular because it is relatively simple to fabricate 4 . However, only a few previous theoretical studies have considered the additional contribution of the free-surface curvature to capillary flow in open rectangular microchannels. These studies primarily focused on the capillary flow of perfectly wetting liquids (liquid equilibrium contact angle $\theta_{0}=0$ ) in microchannels with large aspect ratios $\lambda=\hat{H} / \hat{W}$ (height/width) ${ }^{51,52}$ or reported threedimensional simulations using the volume-of-fluid method to study the effects of gravity on the capillary rise in vertically oriented open rectangular channels ${ }^{53}$.
In open rectangular channels, the free-surface morphology is more complex than in U-shaped and V-shaped channels (Fig. 1). From the channel inlet to the meniscus front $\hat{z}_{M}$ the upper meniscus spans the entire channel width. At the meniscus front, the flow splits into the channel corners provided the liquid equilibrium contact angle $\theta_{0}<\pi / 44^{47}$. This splitting of flow leads to filaments or fingers extending ahead of the meniscus and influencing its propagation. Such a transition is not observed in $\mathrm{U}$ - and V-shaped channels. Note that although the meniscus and fingers are coupled to each other and part of a single free surface, it can be convenient to characterize their behavior separately. For example, Weislogel ${ }^{48}$ modeled the complex free-surface morphology in closed rectangular channels by coupling the advancing meniscus motion using a Lucas-Washburn-type model with the filament propagation using a lubrication-theory-based model.

Recently, Kolliopoulos et al. ${ }^{15}$ used a combination of theory and experiment to examine capillary-flow dynamics in open rectangular microchannels with negligible gravitational effects. Scanning electron microscopy and profilometry were used to highlight the complexity of the free-surface morphology. A self-similar lubrication-theory-based model accounting for this complexity was developed and model predictions were compared to those from the widely used MLW, as well as experimental observations over a wide range of channel aspect ratios $\lambda$ and equilibrium contact angles $\theta_{0}$.

In Fig. 2a, model predictions are compared to experimental observations and the limitations of each model are identified based on the combination of channel aspect ratio $\lambda$ and equilibrium contact angle $\theta_{0}$. Results are superimposed on the free-surface morphology regions identified by Seemann et al. ${ }^{54}$ using a combination of atomic force microscopy experiments and Surface Evolver modeling. For large $\lambda$ the two model predictions are indistinguishable, whereas for smaller $\lambda$ the lubrication-theorybased model agrees better with experiments. The lubricationtheory-based model is in better agreement with experiments at smaller $\theta_{0}$, although as $\theta_{0} \rightarrow \pi / 4$ it fails to account for important axial curvature contributions to the free surface and the agreement worsens. The MLW model agrees with experiments for larger $\lambda$ and larger $\theta_{0}$, where the contributions from the upper liquid-air interface are not as significant since the model assumes 
(a)

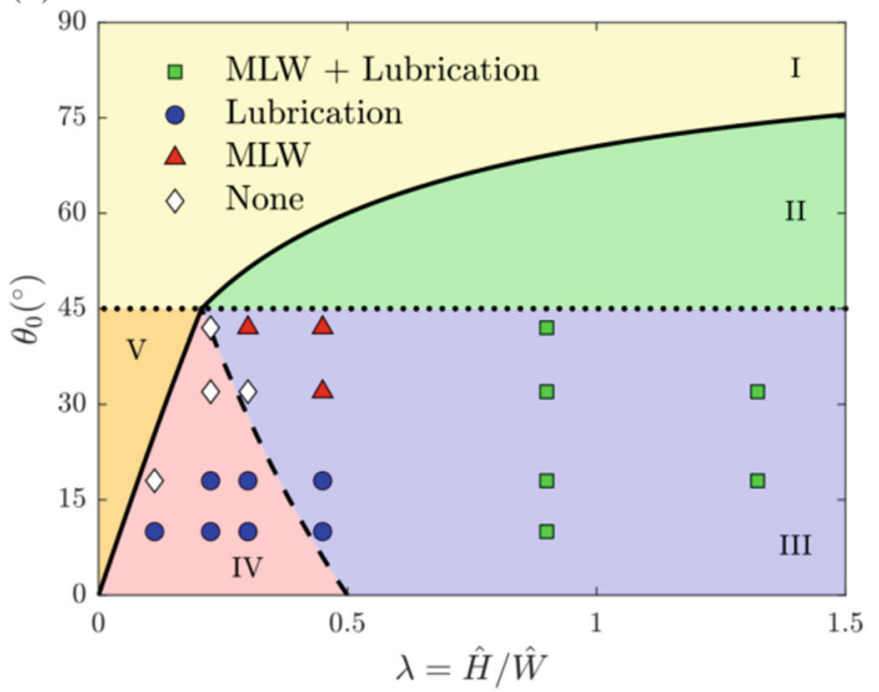

(b)

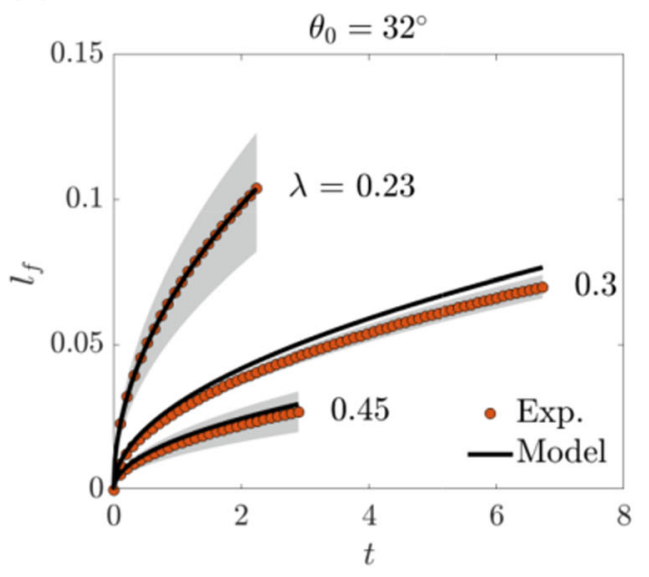

Fig. 2 Free-surface morphology and finger length. a Free-surface morphology diagram as a function of equilibrium contact angle $\theta_{0}$ and channel aspect ratio $\lambda$. Solid symbols represent an agreement between lubrication-theory-based model and MLW model predictions with experiments ${ }^{15}$. The solid line corresponds to the limit where the capillary flow of the meniscus is energetically favorable in an open rectangular channel ${ }^{54}$. The dotted line corresponds to the contact-angle limit below which fingers form ${ }^{47}$. The dashed line corresponds to the critical aspect ratio at which fingers transition from a pinned to an unpinned state at the top of channel sidewall ${ }^{15,54}$. Region I has no capillary flow of the meniscus, region II has no fingers present, region III has fingers that are not pinned to the top of the channel sidewall, region IV has fingers that are pinned to the top of the channel sidewall, and region $\mathrm{V}$ has a capillary flow of fingers but not of the meniscus. $\mathbf{b}$ Effect of aspect ratio $\lambda$ on dimensionless finger length $I_{\mathrm{f}}=\hat{I}_{\mathrm{f}} / \hat{L}$ as a function of dimensionless time $t=\hat{t} \hat{H} \hat{\sigma} / \hat{L}^{2} \hat{\mu}$, where $\hat{L}$ is the channel length ${ }^{15}$. Solid symbols and shaded areas represent the average and range of experimental results, respectively. Solid lines represent lubrication-theorybased model predictions.

a flat upper liquid-air interface. However, for smaller $\lambda$ the agreement worsens because the upper liquid-air interface contribution becomes significant.

The lubrication-theory-based model also quantitatively predicts the dynamics of fingers that extend ahead of the meniscus for a range of channel aspect ratios $\lambda$ (Fig. 2b) and equilibrium contact angles $\theta_{0}$ (not shown here) ${ }^{15}$. These findings elucidate the limitations of the MLW model and demonstrate the importance of accounting for the effects of complex free-surface morphology on capillary-flow dynamics in open rectangular microchannels.

\section{INFLUENCE OF EVAPORATION}

In addition to the complex free-surface morphology, the lack of a top allows evaporation to significantly affect flow if the liquid evaporates. In applications such as microfluidic devices used for diagnostic testing, evaporation can undesirably influence test results ${ }^{5}$. In contrast, in applications such as flexible printed electronics fabrication, evaporation is exploited to print conductive inks on flexible substrates that can be integrated with roll-toroll manufacturing processes, potentially resulting in low-cost and high-throughput device fabrication. A relatively recent example of this is the self-aligned capillarity-assisted lithography for electronics (SCALE) process ${ }^{1-3,55}$.

The SCALE process initially involves using imprint lithography to mold a network of channels and reservoirs into a coated thermoset material (i.e., plastic substrate). An electronic ink is then sequentially deposited in the reservoirs via inkjet printing, and the ink flows into the channels due to capillary forces. During flow, the ink solvent evaporates, leading to the deposition of conductive material onto the channel sidewalls and bottom. This process is repeated using different liquids with successive drying steps to create a variety of electronic devices including resistors, capacitors, and transistors ${ }^{1-3,55}$. The quality and performance of the electronic devices are highly dependent on the simultaneous capillary flow and evaporation of the electronic inks. Hence, developing a fundamental understanding of flow and evaporation in open microchannels is vital to improving and optimizing such applications.

Motivated by thermal management devices such as heat pipes, previous studies have considered the effects of evaporation on steady flow in open rectangular ${ }^{52,56}$ and V-shaped ${ }^{57-60}$ channels. In these studies, the flow reaches a steady state when the capillary flow is balanced by evaporation, assuming an infinite supply of liquid at the channel inlet. The influence of evaporation on flow in V-shaped channels was recently examined theoretically by Gambaryan-Roisman $^{61}$ using a diffusion-limited evaporation model. However, these previous studies focus on the flow of pure liquids, whereas most applications rely on the capillary flow of liquid solutions or colloidal suspensions.

Experiments on the capillary flow of nano- and micro-particle suspensions in open microchannel networks subject to evaporative lithography were conducted by Lone et al. ${ }^{62}$. After the flow reached the end of the channel, the carrier liquid was evaporated, resulting in deposition of the suspended particles on the channel bottom, creating a two-dimensional continuous metal pattern. Although particle suspensions were considered, the meniscus position in the experiments was found to scale as $\hat{z}_{M} \sim \hat{t}^{1 / 2}$, consistent with what is expected for particle-free liquids in the absence of evaporation during flow. The influence of evaporation during flow (i.e., prior to the flow reaching the end of the channel) was not investigated in the experiments.

One of the first studies to examine the effects of evaporation on flow dynamics in open rectangular microchannels with negligible gravitational effects was conducted by Lade et al. ${ }^{37}$. Experiments were conducted using aqueous polymer solutions and electronic inks commonly found in printed electronics fabrication. The rate of evaporation was controlled using a humidity chamber and the meniscus position as a function of time was reported for a range of channel aspect ratios $\lambda$ (height/width) and evaporation rates. Lade et al. also observed nonuniform solute deposition patterns after solvent evaporation, with solute accumulating near the front 
(a)

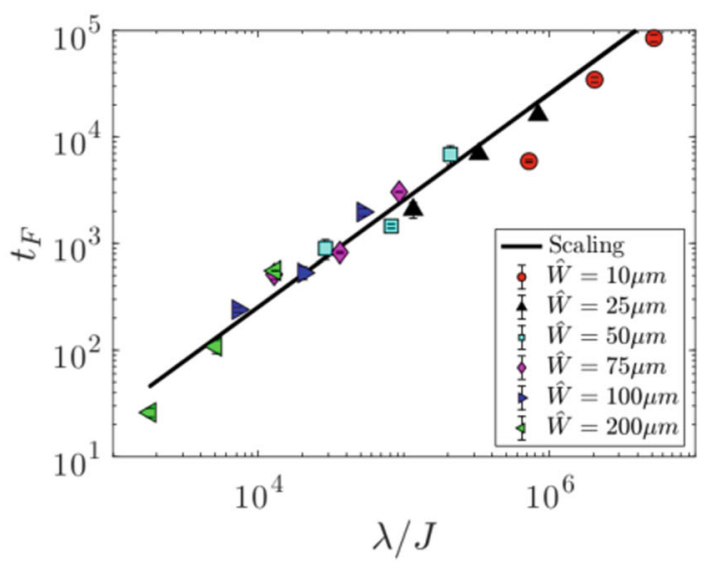

(b)

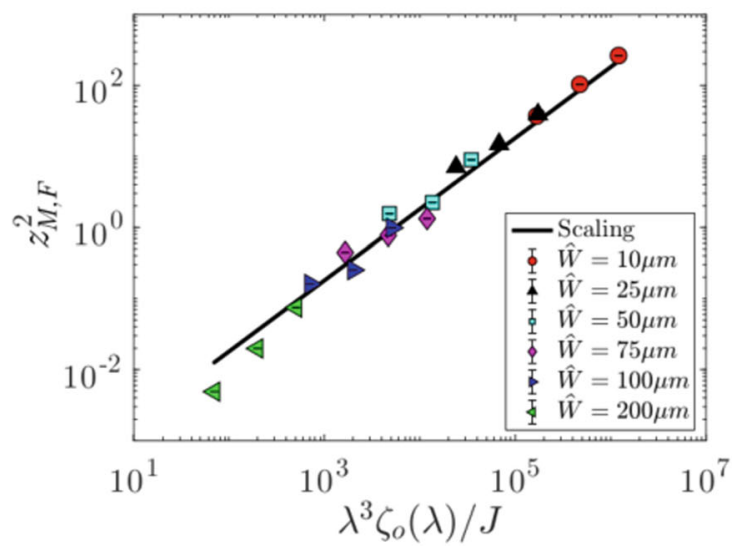

Fig. 3 Scaling relationships. Scaling relationships for (a) dimensionless final time $t_{\mathrm{F}}=\hat{t}_{\mathrm{F}} \hat{\sigma} / \hat{\mu} \hat{W}$ and (b) square of the dimensionless final meniscus position $z_{\mathrm{M}, \mathrm{F}}^{2}=\hat{z}_{\mathrm{M}, \mathrm{F}}^{2} / \hat{W}^{2}$, for different channel widths $\hat{W}^{14}$. The solid lines represent the proposed scaling relationships and the solid symbols represent experimental results by Lade et al. ${ }^{37}$. All channel heights $\hat{H}$ are $46.8 \mu \mathrm{m}$. The channel aspect-ratio function $\zeta_{0}(\lambda)$ can be found in the work of Ouali et al. ${ }^{24}$.

meniscus in a way that resembles the coffee-ring effect commonly observed in evaporating droplets. Strong disagreement was found between the experimental results and the predictions of a MLW model neglecting evaporation, which demonstrated the need for a model that accounts for the effects of evaporation on flow dynamics.

A Lucas-Washburn-type one-dimensional model that incorporates the effects of a concentration-dependent viscosity and uniform evaporation on capillary flow in rectangular microchannels was developed by Kolliopoulos et al. ${ }^{14}$. The model yields predictions of the meniscus position evolution down the microchannel length. Model predictions were compared to the experiments by Lade et al. ${ }^{37}$ involving aqueous poly(vinyl alcohol) solutions by using the evaporation rate as a fitting parameter. The model qualitatively captures the meniscus position evolution for different relative humidities. However, the evaporation rates used to fit the model are $O\left(10-10^{2}\right)$ larger than those obtained from bulk drying experiments. This discrepancy was attributed to assuming a flat free surface and not accounting for axial concentration gradients in the model. Despite this discrepancy, scaling relationships obtained from the model for the dependence of the final meniscus position $z_{\mathrm{M}, \mathrm{F}}$ and total flow time $t_{\mathrm{F}}$ on the channel dimensions and rate of uniform evaporation $J$ are in good agreement with the experimental observations of Lade et al. ${ }^{37}$ (Fig. 3).

Solute concentration gradients arise during the flow of an evaporating liquid solution or colloidal suspension as demonstrated by Lade et al. ${ }^{37}$. These solute concentration gradients are expected to lead to surface-tension gradients, which in turn give rise to Marangoni stresses that act on the liquid to drive flow. Wijnhorst et al. ${ }^{63}$ used experiments to study the effect of surfactants on the dynamics of capillary rise and finger formation in closed rectangular channels and capillary tubes. The addition of surfactant results in reduced meniscus propagation due to the decrease in surface tension, and qualitative differences were observed in finger dynamics compared to pure liquids. The results suggest that surfactants can either enhance and inhibit the finger dynamics depending on the choice of surfactant. While these experiments were conducted using closed channels, where the flow is driven by the pressure gradient caused by the circular-arc meniscus front, for open channels the curvature gradients due to the additional free surface also drive flow. Hence, we expect the addition of surfactants to have a greater influence on flow dynamics in open channels.

\section{CONCLUSIONS}

Exploiting spontaneous capillary flow for the control and transport of liquids is of great importance for numerous in-space and terrestrial applications. While the capillary flow of pure nonvolatile liquids has been extensively investigated, liquid solutions or colloidal suspensions with volatile components or surfactants have received less attention despite many applications relying on such liquids. New insights into the effects of evaporation and surfactants on the capillary flow of multicomponent liquids have practical implications for numerous applications, such as lab-on-a-chip devices, heat pipes, propellant management devices in spacecrafts, paper-based microfluidics, fuel cells, porous-media flows, and fabrication of flexible printed electronics. Comprehensive physical understanding of this topic will likely involve a close interplay between experiments, analytical and semi-analytical models, and direct numerical simulations.

\section{DATA AVAILABILITY}

All data analyzed during this study are included in this published article.

\section{CODE AVAILABILITY}

No custom computer codes or algorithms were used to generate new results for this paper.

Received: 21 June 2021; Accepted: 3 November 2021; Published online: 09 December 2021

\section{REFERENCES}

1. Cao, M., Jochem, K. S., Hyun, W.-J., Francis, L. F. \& Frisbie, C. D. Self-aligned inkjet printing of resistors and low-pass resistor-capacitor filters on roll-to-roll imprinted plastics with resistances ranging from 10 to $10^{6} \Omega$. Flex. Print. Electron. 3, 045003 (2018).

2. Jochem, K. S., Suszynski, W. J., Frisbie, C. D. \& Francis, L. F. High-resolution, highaspect-ratio printed and plated metal conductors utilizing roll-to-roll microscale UV imprinting with prototype imprinting stamps. Ind. Eng. Chem. Res. 57, 16335-16346 (2018).

3. Jochem, K. S. et al. Self-aligned capillarity-assisted printing of high aspect ratio flexible metal conductors: optimizing ink flow, plating, and mechanical adhesion. Ind. Eng. Chem. Res. 59, 22107-22122 (2020).

4. Olanrewaju, A., Beaugrand, M., Yafia, M. \& Juncker, D. Capillary microfluidics in microchannels: from microfluidic networks to capillaric circuits. Lab Chip 18, 2323-2347 (2018). 
5. Oliveira, N. M., Vilabril, S., Oliveira, M. B., Reis, R. L. \& Mano, J. F. Recent advances on open fluidic systems for biomedical applications: a review. Mater. Sci. Eng. $C$ 97, 851-863 (2019).

6. Faghri, A. Heat Pipe Science and Technology (Global Digital Press, 1995).

7. Levine, D. et al. Surface tension and contact angle analysis with design of propellant measurement apparatus. J. Propul. Power 31, 429-443 (2015).

8. Martinez, A. W. et al. Simple telemedicine for developing regions: camera phones and paper-based microfluidic devices for real-time, off-site diagnosis. Anal. Chem. 80, 3699-3707 (2008)

9. del Torno-de Román, L. et al. Improved performance of a paper-based glucose fuel cell by capillary induced flow. Electrochim. Acta 282, 336-342 (2018).

10. Marmur, A. \& Cohen, R. D. Characterization of porous media by the kinetics of liquid penetration: the Vertical Capillaries Model. J. Colloid Interface Sci. 189, 299-304 (1997)

11. Cai, J. et al. Lucas-Washburn equation-based modeling of capillary-driven flow in porous systems. Langmuir 37, 1623-1636 (2021).

12. Rollins, J. R., Grove, R. K. \& Jaekle Jr., D. E. Twenty-three years of surface tension propellant management system-design, development, manufacture, test, and operation. In AIAA Paper, 85-1199 (1985).

13. McCraney, J., Weislogel, M. \& Steen, P. OpenFOAM simulations of late stage container draining in microgravity. Fluids 5, 207 (2020).

14. Kolliopoulos, P., Jochem, K. S., Lade Jr., R. K., Francis, L. F. \& Kumar, S. Capillary flow with evaporation in open rectangular microchannels. Langmuir 35 8131-8143 (2019).

15. Kolliopoulos, P. et al. Capillary-flow dynamics in open rectangular microchannels. J. Fluid Mech. 911, A32 (2021)

16. Lucas, R. Ueber das Zeitgesetz des kapillaren Aufstiegs von Flüssigkeiten. Kolloid Z. 23, 15-22 (1918)

17. Washburn, E. W. The dynamics of capillary flow. Phys. Rev. 17, 273 (1921).

18. Quéré, D. Inertial capillarity. Europhys. Lett. 39, 533-538 (1997).

19. Rideal, E. K. On the flow of liquids under capillary pressure. Philos. Mag. 44, 1152-1159 (1922).

20. Bosanquet, C. H. LV. On the flow of liquids into capillary tubes. Philos. Mag. 45 525-531 (1923)

21. Fries, N. \& Dreyer, M. The transition from inertial to viscous flow in capillary rise. J. Colloid Interface Sci. 327, 125-128 (2008).

22. Siebold, A., Nardin, M., Schultz, J., Walliser, A. \& Oppliger, M. Effect of dynamic contact angle on capillary rise phenomena. Colloids Surf. A 161, 81-87 (2000).

23. Popescu, M. N., Ralston, J. \& Sedev, R. Capillary rise with velocity-dependent dynamic contact angle. Langmuir 24, 12710-12716 (2008).

24. Ouali, F. F. et al. Wetting considerations in capillary rise and imbibition in closed square tubes and open rectangular cross-section channels. Microfluid. Nanofluid. 15, 309-326 (2013)

25. Xing, H., Cheng, J. \& Zhou, C. Effect of gradient wettability on capillary imbibition in open semicircular copper channel. Phys. Fluids 32, 112004 (2020).

26. O'Loughlin, M., Priest, C., Popescu, M. N. \& Ralston, J. Patterning of wettability for controlling capillary-driven flow in closed channels. J. Appl. Mech. 402, 259-266 (2013).

27. Baret, J.-C., Decré, M., Herminghaus, S. \& Seemann, R. Transport dynamics in open microfluidic grooves. Langmuir 23, 5200-5204 (2007).

28. Shan, F., Chai, Z. \& Shi, B. A theoretical study on the capillary rise of nonNewtonian power-law fluids. Appl. Math. Model. 81, 768-786 (2020).

29. Berli, C. L. A. \& Urteaga, R. Asymmetric capillary filling of non-Newtonian power law fluids. Microfluid. Nanofluid. 17, 1079-1084 (2014).

30. Fisher, L. R. \& Lark, P. D. An experimental study of the Washburn equation for liquid flow in very fine capillaries. J. Colloid Interface Sci. 69, 486-492 (1979).

31. Ichikawa, N. \& Satoda, Y. Interface dynamics of capillary flow in a tube under negligible gravity condition. J. Colloid Interface Sci. 162, 350-355 (1994).

32. Ichikawa, N., Hosokawa, K. \& Maeda, R. Interface motion of capillary-driven flow in rectangular microchannel. J. Colloid Interface Sci. 280, 155-164 (2004).

33. Heshmati, M. \& Piri, M. Experimental investigation of dynamic contact angle and capillary rise in tubes with circular and noncircular cross sections. Langmuir $\mathbf{3 0}$ 14151-14162 (2014).

34. Miranda, A. M., Menezes-Sobrinho, I. L. \& Couto, M. S. Spontaneous imbibition experiment in newspaper sheets. Phys. Rev. Lett. 104, 086101 (2010).

35. Yang, D., Krasowska, M., Priest, C., Popescu, M. N. \& Ralston, J. Dynamics of capillary-driven flow in open microchannels. J. Phys. Chem. C 115, 18761-18769 (2011)

36. Sowers, T. W., Sarkar, R., Prameela, S. E., Izadi, E. \& Rajagopalan, J. Capillary driven flow of polydimethylsiloxane in open rectangular microchannels. Soft Matter 12 5818-5823 (2016).

37. Lade Jr., R. K., Jochem, K. S., Macosko, C. W. \& Francis, L. F. Capillary coatings: flow and drying dynamics in open microchannels. Langmuir 34, 7624-7639 (2018).
38. Chen, T. Capillary force-driven fluid flow of a wetting liquid in open grooves with different sizes. In Fourteenth Intersociety Conference on Thermal and Thermomechanical Phenomena in Electronic Systems (ITherm), 388-396 (IEEE, 2014).

39. Rye, R. R., Yost, F. G. \& O'Toole, E. J. Capillary flow in irregular surface grooves. Langmuir 14, 3937-3943 (1998).

40. Mann Jr., J. A., Romero, L. A., Rye, R. R. \& Yost, F. G. Flow of simple liquids down narrow V grooves. Phys. Rev. E 52, 3967-3972 (1995).

41. Rye, R. R., Mann Jr., J. A. \& Yost, F. G. The flow of liquids in surface grooves Langmuir 12, 555-565 (1996).

42. Yost, F. G., Rye, R. R. \& Mann Jr., J. A. Solder wetting kinetics in narrow v-grooves. Acta Mater. 45, 5337-5345 (1997).

43. Schift, H. et al. Quantitative analysis of the molding of nanostructures. J. Vac. Sci. Technol. B 18, 3564 (2000).

44. Berthier, J., Gosselin, D. \& Berthier, E. A generalization of the Lucas-Washburn-Rideal law to composite microchannels of arbitrary cross section. Microfluid. Nanofluid.19, 497-507 (2015).

45. Romero, L. A. \& Yost, F. G. Flow in an open channel capillary. J. Fluid Mech. 322 109-129 (1996).

46. Weislogel, M. M. \& Lichter, S. Capillary flow in an interior corner. J. Fluid Mech. 373, 349-378 (1998)

47. Concus, P. \& Finn, R. On the behaviour of a capillary surface in a wedge. Proc. Natl Acad. Sci. USA 63, 292-299 (1969).

48. Weislogel, M. M. Compound capillary rise. J. Fluid Mech. 709, 622-647 (2012).

49. Ponomarenko, A., Quéré, D. \& Clanet, C. A universal law for capillary rise in corners. J. Fluid Mech. 666, 146-154 (2011).

50. Gurumurthy, V. T., Rettenmaier, D., Roisman, I. V., Tropea, C. \& Garoff, S. Computations of spontaneous rise of a rivulet in a corner of a vertical square capillary. Colloids Surf. A 544, 118-126 (2018).

51. Tchikanda, S. W., Nilson, R. H. \& Griffiths, S. K. Modeling of pressure and sheardriven flows in open rectangular microchannels. Int. J. Heat Mass Transf. 47, 527-538 (2004).

52. Nilson, R. H., Tchikanda, S. W., Griffiths, S. K. \& Martinez, M. J. Steady evaporating flow in rectangular microchannels. Int. J. Heat Mass Transf. 49, 1603-1618 (2006).

53. Gurumurthy, V. T., Roisman, I. V., Tropea, C. \& Garoff, S. Spontaneous rise in open rectangular channels under gravity. J. Colloid Interface Sci. 527, 151-158 (2018).

54. Seemann, R., Brinkmann, M., Kramer, E. J., Lange, F. F. \& Lipowsky, R. Wetting morphologies at microstructured surfaces. Proc. Natl Acad. Sci. USA 102 1848-1852 (2005).

55. Mahajan, A. et al. A self-aligned strategy for printed electronics: exploiting capillary flow on microstructured plastic surfaces. Adv. Electron. Mater. 1, 1500137 (2015).

56. Xia, Z. Z., Yang, G. Z. \& Wang, R. Z. Capillary-assisted flow and evaporation inside circumferential rectangular micro groove. Int. J. Heat Mass Transf. 52, 952-961 (2009).

57. Markos, M., Ajaev, V. S. \& Homsy, G. M. Steady flow and evaporation of a volatile liquid in a wedge. Phys. Fluids 18, 092102 (2006).

58. Suman, B. \& Hoda, N. Effect of variations in thermophysical properties and design parameters on the performance of a V-shaped micro grooved heat pipe. Int. J. Heat Mass Transf. 48, 2090-2101 (2005).

59. Peterson, G. P. \& Ma, H. B. Theoretical analysis of the maximum heat transport in triangular grooves: a study of idealized micro heat pipes. J. Heat Transf. 118 731-739 (1996).

60. Khrustalev, D. \& Faghri, A. Thermal analysis of a micro heat pipe. J. Heat Transf. 116, 189-198 (1994).

61. Gambaryan-Roisman, T. Simultaneous imbibition and evaporation of liquids on grooved substrates. Interfacial Phenom. Heat Transf. 7, 239-253 (2019).

62. Lone, S., Zhang, J. M., Vakarelski, I. U., Li, E. Q. \& Thoroddsen, S. T. Evaporative lithography in open microfluidic channel networks. Langmuir 33, 2861-2871 (2017).

63. Wijnhorst, R., de Goede, T. C., Bonn, D. \& Shahidzadeh, N. Surfactant effects on the dynamics of capillary rise and finger formation in square capillaries. Langmuir 36, 13784-13792 (2020).

\section{ACKNOWLEDGEMENTS}

This work was supported by the National Science Foundation (NSF) under Grant Nos. CMMI-1634263 and CMMI-2038722. We thank Lorraine Francis, Dan Frisbie, Krystopher Jochem, Bob Lade, and Wieslaw Suszynski for helpful discussions. We dedicate this article to the memory of Paul Steen, who provided important encouragement and inspiration for S.K.'s research efforts in interfacial fluid mechanics.

\section{AUTHOR CONTRIBUTIONS}

Both P.K. and S.K. participated in the writing and editing of this manuscript. 


\section{COMPETING INTERESTS}

The authors declare no competing interests.

\section{ADDITIONAL INFORMATION}

Correspondence and requests for materials should be addressed to Satish Kumar.

Reprints and permission information is available at http://www.nature.com/ reprints

Publisher's note Springer Nature remains neutral with regard to jurisdictional claims in published maps and institutional affiliations.
Open Access This article is licensed under a Creative Commons Attribution 4.0 International License, which permits use, sharing, adaptation, distribution and reproduction in any medium or format, as long as you give appropriate credit to the original author(s) and the source, provide a link to the Creative Commons license, and indicate if changes were made. The images or other third party material in this article are included in the article's Creative Commons license, unless indicated otherwise in a credit line to the material. If material is not included in the article's Creative Commons license and your intended use is not permitted by statutory regulation or exceeds the permitted use, you will need to obtain permission directly from the copyright holder. To view a copy of this license, visit http://creativecommons. org/licenses/by/4.0/.

(c) The Author(s) 2021 